\title{
A Tangible User Interface Using Spatial Augmented Reality
}

\author{
Leith K. Y. Chan* Henry Y. K. Lau \\ Department of Industrial and Manufacturing Systems Engineering \\ The University of Hong Kong, Hong Kong
}

\begin{abstract}
In this paper, we describe the novel implementation of a tangible user interface framework, namely the MagicPad, inspired by the concept of Spatial Augmented Reality. By using an Infrared pen with any flat surface, such as a paper pad that receives projected images from a projector, a user is able to perform a variety of interactive visualization and manipulation in the 3D space. Two implementations using the MagicPad framework are presented, which include the magic lenses like interface inside a CAVE-like system and a virtual book in an art installation.
\end{abstract}

KEYWORDS: augmented reality, 3D user interface.

\section{INTRODUCTION AND BACKGROUND}

With the reduction of the cost of computer hardware and the rapid development of PC based graphics processors, the Virtual Reality environment is becoming more affordable and popular. Large high-resolution displays have been widely applied in various domains [1]. These Virtual Reality systems give the user the immersive experience of entering a computer generated virtual world with Augmented Reality (AR) rather than immersing a person into a completely synthetic world, it attempts to embed synthetic supplements into the real environment [2]. Augmented Reality opens up a new area of user interface design since all real and tangible objects with embedded virtual entities can be treated as a tool or interface to navigate around and manipulate the objects in the virtual world. A number of studies have been done to investigate the possibility of using AR to improve the user interface inside a virtual environment.

In this paper, we propose the design and describe the implementation of an intuitive user interface framework, known as MagicPad. The idea of the MagicPad comes from the concept of the Projection-Based Spatial Augmented Reality. In projectorbased augmentation, the user's physical environment is augmented with images that are integrated directly into the user's environment [3]. The user and the augmented physics objects coexist in the same 3D virtual environment. Naturally, the augmented objects have great potential to be the ideal interface between the user and the virtual environment [4].

\section{Design Concept}

The MagicPad interface mainly consists of one or more flat surfaces, which can be a white cardboard or sketch book pages. The white surface (MagicPad) acts as a display to receive the image from the ceiling mounted projector (Figure 1). The position

*e-mail: 1kychan@hku.hk

†e-mail: hyklau@hku.hk

IEEE Symposium on 3D User Interfaces 2010

20-21 March, Waltham, Massachusetts, USA

978-1-4244-6847-8/10/\$26.00 C2010 IEEE and orientation of the MagicPad are tracked by an optical tracking system. When the MagicPad moves, the image projected by the projector will be updated to match the motion of the MagicPad. This results in the illusion that the image is glued onto the MagicPad's surface without relative movement. In addition, the user can use an infrared pen, which is also being tracked, to interact with the MagicPad. Since the MagicPad and the infrared pen are light weight devices, the user can easily hold them with his/her hands and perform series of $3 \mathrm{D}$ interactions with the virtual environment. In fact, the original innovation of the MagicPad is aimed to provide the similar experience of using a pen and paper. With this familiar interface tools, we target to allow a layman user, even without prior experience in using VR interfaces, to be able to use the MagicPad right away without much training.

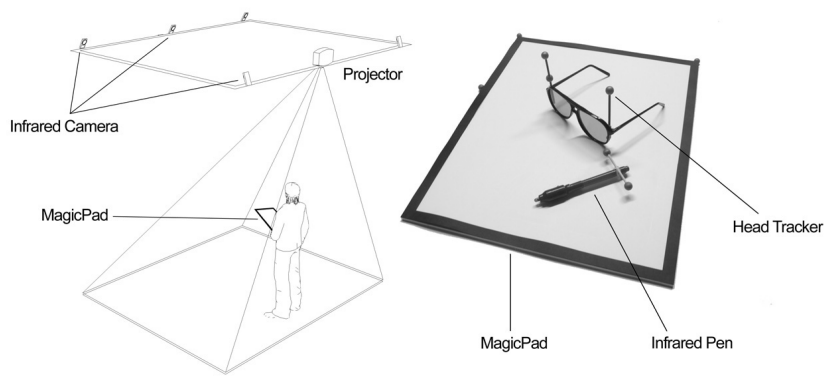

Figure 1. The design concept and hardware configuration of the MagicPad Interface

\section{SYSTEM IMPLEMENTATION}

In order to test drive the MagicPad framework, two MagicPad applications have been developed to study the performance and applicability of the framework. These applications are the implementation of the MagicPad interface inside a CAVE-like system and an art installation.

\subsection{MagicPad interface inside a CAVE-like system}

In the field of 3D scientific visualization and human-computer interface, researchers have developed techniques to overcome the obstacles of displaying and manipulation of complex data. Among them, an interface technique called Magic Lenses [5] has been introduced. Since then, many new user interfaces inspired by this technique have been developed. Some of them incorporated the idea of augmented reality to develop a Magic Lens framework for the augmented virtual environment[6-7]. In our implementation, we use the MagicPad infrastructure to create a Magic Lenses inspired user interface to navigate the virtual environment inside a CAVE-like system, namely the imseCAVE, developed by the Department of Industrial and Manufacturing Systems Engineering, The University of Hong Kong.

Inside the imseCAVE, an additional projector is mounted on the top to project images on the MagicPad, which acts as an addition window to examine and interact with the virtual world. Since the MagicPad has two sides and only one of them can receive the 
projected image at a given moment, it is natural to assign different interface behaviors to each side. Therefore, the MagicPad has two modes: Window mode and Overview mode.

- Window Mode: In the window mode, the MagicPad acts as an additional viewing window to the virtual world. The system renders the image based on the perspective of the user's viewpoint on the MagicPad in real-time. As a result, the user has the illusion of seeing through the MagicPad. This virtual window offers three rendering modes to help the user to examine the objects in the virtual world. They are (i) Normal Mode, (ii) Wireframe Mode and, (iii) Magnifying Mode. The user can simply flip the MagicPad back and forth momentarily to switch between these modes. In addition, the user can use the infrared pen to write on the MagicPad in order to move to any directions in the $3 \mathrm{D}$ space or turning the view point.

- Overview Mode: the MagicPad shows the plan view of the virtual world with the imseCAVE at the lower center. It allows the user to navigate from a third person viewpoint in a larger scale so that the user is able to relocate itself to another location quickly and effectively.
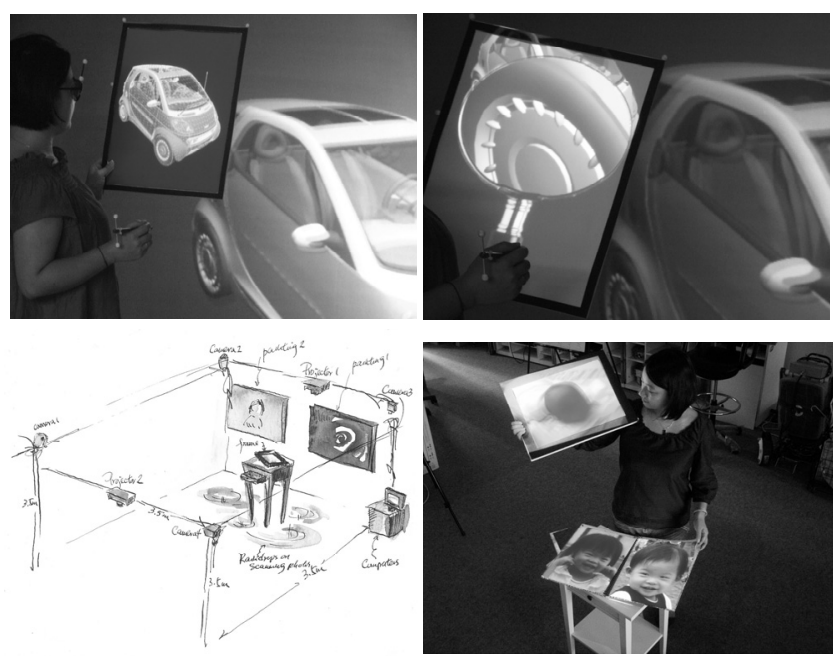

Figure 2. Examination of car model (top) inside the imseCAVE and an art installation (bottom) using the MagicPad Interface

\section{2 'Head Profile' - An Art Installation}

The MagicPad framework has also been deployed in creating an art installation named "Head Profiles", as illustrated in Figure 2. "Head Profiles" is a mélange of traditional art medium and interactive computer graphics. The artist intends to share the amazing journey of her motherhood by the art piece. Two traditional acrylic paintings in the background depict the different stages of her child: a 2 years old portrait and an ultrasound scan of the fetus. The MagicPad becomes a photo frame and a sketch book to be handled by spectators. The photo frame displays the ultrasound scan of a fetus when it is rested on the table. When the photo frame is held up in the air, it starts to display a video clip of the child first raising her head on her own at the age of one month old. The sketch book has 4 pages with each page displaying a picture of the child's face taken at different ages. The images displayed on the page change according to the distance between the sketch book and the two background paintings.

\section{Advantages and Limitations}

The MagicPad interface is designed and developed based on the Projector-based Spatial Augmented Reality and therefore shares similar benefits of spatially immersive displays [8]. The key benefit of the MagicPad is that a user does not need to wear a head-mount display or use a monitor to see the augmented images. In addition, the lightweight MagicPad provides a tangible, familiar and natural user interface between the user and the computer system. Our preliminary subject test also shows that users were able to discover and master the different operations supported by the interface within a short period of time without detail initial explanation of its usage. This is particular handy in the case of the art installation, where the visitors are free to explore by themselves. On the other hand, the MagicPad interface also inherits the technical limitations of Spatial Augmented Reality including limited resolution and depth of field of the projector, problems of casting shadow and poor image formation when the projection angle is too steep. Some of these problems can be solved by adding more projectors to project images from different angles; though achieving seamless imagery with multiple projectors can be a challenging undertaking. In addition, the latency (or lag) caused by the time taken from the tracking system, the networking communication and the image rendering also affects how well the image is glued onto a moving MagicPad's surface. In our system, the latency is around $80 \mathrm{~ms}$. For an average user, this latency results in the drifting of the image by around $5 \mathrm{~cm}$ for slow movement and $30 \mathrm{~cm}$ for fast movement of the MagicPad.

\section{CONCLUSION}

In this paper, we have described the design, architecture and implementation of the MagicPad framework. Two applications based on this framework have been presented. Although further user studies have to be done to investigate the capability of the MagicPad interface, preliminary evaluations have shown that this interface enriches the user experience and enhances the effectiveness of human-computer interface. With the declining price of optical tracking systems and emergence of low cost optical tracking solutions, spatial augmented reality tools and applications are more easily reaching the general public, and hence starting to reveal its potential in a variety of application domains, including art, design and entertainment.

\section{REFERENCES}

[1] T. Ni, et al., "A Survey of Large High-Resolution Display Technologies, Techniques, and Applications," IEEE VR, 2006.

[2] O. Bimber and R. Raskar, "Modern approaches to augmented reality," ACM SIGGRAPH Courses, Los Angeles, California, 2005.

[3] R. Raskar, et al., "Multi-projector displays using camera-based registration," IEEE Visualization, San Francisco, California, United States, 1999.

[4] R. Raskar and K.-L. Low, "Interacting with spatially augmented reality," Computer Graphics, Virtual Reality and Visualisation, Camps Bay, Cape Town, South Africa, 2001

[5] E. A. Bier, et al., "Toolglass and magic lenses: the see-through interface," Computer Graphics and Interactive Techniques, Anaheim, CA, 1993

[6] L. D. Brown and H. Hua, "Magic Lenses for Augmented Virtual Environments," In IEEE Computer Graphics and Applications., vol. 26, pp. 64-73, 2006

[7] S. S. Martin Spindler, Raimund Dachselt, "PaperLens: Advanced Magic Lens Interaction Above the Tabletop," Interactive Tabletops and Surfaces, Banff, Canada, 2009.

[8] E. Lantz, "The future of virtual reality: head mounted displays versus spatially immersive displays (panel)," Computer Graphics and Interactive Techniques, 1996. 